\section{Integrative Medicine \\ International}

\title{
The Treatment Strategies for Neurodegenerative Diseases by Integrative Medicine
}

\author{
Xiangjun Chen ${ }^{a}$ Weidong Pan ${ }^{b}$ \\ Departments of Neurology, ${ }^{a}$ Huashan Hospital Affiliated to Fudan University, and \\ bShuguang Hospital Affiliated to Shanghai University of Traditional Chinese Medicine, \\ Shanghai, China
}

Acute and chronic neurodegenerative diseases, including prion disease, frontotemporal dementia, Pick's disease, progressive supranuclear palsy, spinocerebellar ataxias, brain trauma, amyotrophic lateral sclerosis (ALS), Huntington's disease, Alzheimer's disease, and Parkinson's disease (PD), are illnesses associated with high morbidity and mortality rates [1, 2]. A characteristic of many neurodegenerative diseases is progressive neuronal cell death [3]. During the past decade, considerable progress has been made in understanding the process of cell death [4]. The symptoms and the exacerbations of these diseases are much different according to their specific pathways of cell death, and having their own mechanisms of cell death leads to novel therapeutic strategies.

Today, there is no treatment that can cure degenerative diseases, but we have many symptomatic treatments. There are some advantages of Western medicines for these diseases, such as dopaminergic treatments for PD and movement disorders [5], cholinesterase inhibitors for cognitive disorders [6], antipsychotic drugs for behavioral and psychological symptoms of dementia [7], analgesic drugs for pain [8], anti-inflammatories for infections [9], and even the use of deep brain stimulation to stop tremor and refractory movement disorders [10]. Researchers have also aimed to produce medicines to slow the development of diseases, such as Riluzole for ALS, cerebellar ataxia and Huntington's disease [11-13], NSAIDs (nonsteroidal anti-inflammatory drugs) for Alzheimer's disease [14], and caffein A2A receptor antagonists and CERE-120 (adeno-associated virus serotype 2-neurturin) for the neuroprotection of PD $[15,16]$. However, we have not yet progressed very well; there still remain too many problems to administrate the progressive and serious symptoms of these diseases [5].

Including Western medicine and traditional medicine, integrative medicine [17] has its advantages in treating degenerative diseases. Integrative medicine aims to be 'integrative' or 'whole'; it does not only treat the acute symptoms of these diseases, but it also considers all of the related symptoms and tries to improve the quality of activity of daily life (ADL) for the patients [18]. Non-motor disorders is one refractory symptom of PD, such as sleep disorders, 
autonomic symptoms, neuropsychiatric symptoms, gastrointestinal symptoms, sensory symptoms, and other symptoms including non-motor fluctuations and fatigue [8]. Progressive ALS exhibits many symptoms and influences the ADL of ALS patients significantly. And, at later stages, as with many other degenerative diseases, patients will suffer from inflammation, expiratory dyspnea, dysphagia, akinesia, etc.

Including Western medicine, other integrative medicines have also demonstrated excellent results for the serious problems of many degenerative diseases. Pan et al. [19-21] demonstrated that traditional Chinese medicine (TCM) together with Western medicine can improve sleep disorders and other non-motor disorders of PD patients. Chua et al. [22] described the same effects of TCM on the quality of life when treating dyskinesias and nonmotor symptoms in Chinese PD patients through a double-blind clinical study. Lloret et al. [23] observed that Ayurveda medicine has a long history for the treatment of PD with fewer side effects. Pan et al. [24] indicated in one of their studies that TCM can improve the behavioral and psychological symptoms of dementia in Alzheimer's disease. In another study [25], they also reported that TCM is not less effective than Riluzole in treating ALS, and moreover that TCM decoction can slow the development of ALS even more than Riluzole. Li et al. [26] showed that the Tai Chi Quan can increase the stability of most PD patients. Most researchers aim at improving the ADL of degenerative disease patients by complementary and alternative medicine or integrative medicines $[27,28]$. In recent years, researchers have also focused on increasing the ability of reserve to protect the mobility of degenerative disease patients by integrative medicine [29]; we expect they will make some progress.

There are no effective treatments for degenerative diseases in modern society, but we can use Western medicine to deal with the acute disorders or symptoms and use the advantages of other integrative treatments to assist Western medicine in improving the ADL of the patients. Integrative medicine can, sometimes, exhibit protective effects or slow the morbidity of these diseases. We believe that with the development of integrative medicine and modern science, the former will increasingly take a more and more important role in the treatment of degenerative diseases.

\section{Acknowledgments}

This study was sponsored and supported by the National Natural Science Foundation of China (81373619).

\section{Disclosure Statement}

The authors declare that there are no conflicts of interest regarding the publication of this article.

\section{References}

1 Prusiner SB: Shattuck lecture - neurodegenerative diseases and prions. N Engl J Med 2001;344:1516-1526.

2 Friedlander RM: Apoptosis and caspases in neurodegenerative diseases. N Engl J Med 2003;348:1365-1375.

3 Yuan J, Yankner BA: Apoptosis in the nervous system. Nature 2000;407:802-809.

4 Hengartner MO: The biochemistry of apoptosis. Nature 2000;407:770-776.

5 Mizuno Y: Recent research progress in and future perspective on treatment of Parkinson's disease. Integr Med Int 2014;1:67-79.

6 Crane PK, Doody RS: Donepezil treatment of patients with MCI: a 48-week randomized, placebo-controlled trial. Neurology 2009;73:1514-1515.

7 Desai AK, Grossberg GT: Diagnosis and treatment of Alzheimer's disease. Neurology 2005;64(suppl 3):S34S39. 
-8 Chaudhuri KR, Schapira AHV: Non-motor symptoms of Parkinson's disease: dopaminergic pathophysiology and treatment. Lancet Neurol 2009;8:464-474.

-9 Tizabi Y, Hurley LL, Qualls Z, Akinfiresoye L: Relevance of the anti-inflammatory properties of curcumin in neurodegenerative diseases and depression. Molecules 2014;19:20864-20879.

10 Okun MS: Deep-brain stimulation - entering the era of human neural-network modulation. N Engl J Med 2014. 371:1369-1373.

11 Traynor BJ, Bruijn L, Conwit R, et al: Neuroprotective agents for clinical trials in ALS: a systematic assessment Neurology 2006;67:20-27.

12 Ristori G, Romano S, Visconti A, et al: Riluzole in cerebellar ataxia: a randomized, double-blind, placebocontrolled pilot trial. Neurology 2010;74:839-845.

-13 Armstrong MJ, Miyasaki JM; American Academy of Neurology: Evidence-based guideline: pharmacologic treatment of chorea in Huntington disease: report of the guideline development subcommittee of the American Academy of Neurology. Neurology 2012;79:597-603.

-14 Stewart WF, Kawas C, Corrada M, Metter EJ: Risk of Alzheimer's disease and duration of NSAID use. Neurology 1997;48:626-632.

15 Schwarzschild MA, Xu K, Oztas E, Petzer JP: Neuroprotection by caffeine and more specific A2A receptor antagonists in animal models of Parkinson's disease. Neurology 2003;61(suppl 6):S55-S61.

-16 Marks WJ Jr, Ostrem JL, Verhagen L, et al: Safety and tolerability of intraputaminal delivery of CERE-120 (adeno-associated virus serotype 2-neurturin) to patients with idiopathic Parkinson's disease: an open-label, phase I trial. Lancet Neurol 2008; 7:400-408.

17 Pan W, Zhou H: Inclusion of integrative medicine in clinical practice. Integr Med Int 2014;1:1-4.

18 Pan W, Zhou H: Integrative medicine: a paradigm shift in clinical practice. Int J Integr Med 2013;1:21.

19 Pan W, Liu J, Wang Q, et al: Clinical study on chronic pain in Parkinson's disease patients in Shanghai, China. Integr Med Int 2014;1:93-101.

20 Pan W, Kwak S, Liu Y, et al: Traditional Chinese medicine improves activities of daily living in Parkinson's disease. Parkinson Dis 2011;2011:789506.

-21 Pan W, Kwak S, Liu Y, et al: A compound belonging to traditional Chinese medicine improves nocturnal activity in Parkinson's disease. Sleep Med 2011;12:307-308.

-22 Chua K, Gao J, Kum W-F, Li M: The impact of treatment-related dyskinesias and non-motor symptoms on the quality of life in Chinese patients with idiopathic Parkinson's disease. Int J Integr Med 2013;1:31.

23 Lloret SP, Rey MV, Rascol O: Ayurveda medicine for the treatment of Parkinson's disease. Int J Integr Med 2013;1:6.

24 Pan W, Wang Q, Kwak S, et al: Shen-Zhi-Ling oral liquid improves behavioral and psychological symptoms of dementia in Alzheimer's disease. Evid Based Complement Alternat Med 2014;2014:913687.

25 Pan W, Su X, Bao J, et al: Open randomized clinical trial on JWSJZ decoction for the treatment of ALS patients. Evid Based Complement Alternat Med 2013;2013:347525.

-26 Li F, Harmer P, Fitzgerald K, et al: Tai chi and postural stability in patients with Parkinson's disease. N Engl J Med 2012;366:511-519.

27 Kline KL: Complementary and alternative medicine for neurologic disorders. Clin Tech Small Anim Pract 2002; $17: 25-33$.

28 Kum WF, Durairajan SS, Bian ZX, et al: Treatment of idiopathic Parkinson's disease with traditional Chinese herbal medicine: a randomized placebo-controlled pilot clinical study. Evid Based Complement Alternat Med 2011;2011:724353.

29 Jiang W, Pan W: Integrative cognitive reserve. Integr Med Int 2014;3:190-197. 